\title{
Quantitative Monitoring of the Fermentation Process of a Barley Malt Mash by Benchtop ${ }^{1} \mathrm{H}$ NMR Spectroscopy
}

\author{
Pia Burkhardtsmaier ${ }^{1} \cdot$ Kristina Pavlovskaja $^{1}$ - Dennis Maier ${ }^{1}$. Stephanie Schäfer ${ }^{1}$. Ulrike Salat ${ }^{1,2}$. \\ Magnus S. Schmidt ${ }^{1,3}$ (iD)
}

Received: 15 October 2020 / Accepted: 9 February 2021 / Published online: 20 February 2021

(C) The Author(s) 2021

\begin{abstract}
In order to investigate benchtop NMR spectroscopy as a monitoring tool for fermentation processes, we used a barley malt mash and took various samples over time and analysed them by NMR spectroscopy with 3-(trimethylsilyl)-2,2,3,3tetradeuteropropionic acid sodium salt (TSP-d4) as an internal standard for the quantification of ethanol and validated the results by two different enzymatic standard test kits for ethanol analysis. We could show that the results between NMR spectroscopy and test kits were consistent with NMR having a much lower standard deviation. Finally, we discussed the applicability of the method as well as the possibility to quantify various other substances.
\end{abstract}

Keywords Ethanol $\cdot$ Glucose $\cdot$ Maltose $\cdot \mathrm{NMR}$ spectroscopy $\cdot$ Fermentation $\cdot$ Enzymatic test tits

\section{Introduction}

The fermentation of natural products for the production of alcoholic drinks and spirits has a history dating back in time as long as the history of humankind itself (Gately 2009; Hames 2014; Phillips 2014). And though the experience of the manufacturer has an important influence on the results of

Magnus S. Schmidt

magnus.schmidt@hs-furtwangen.de

Pia Burkhardtsmaier

p.burkhardtsmaier@hs-furtwangen.de

Kristina Pavlovskaja

kristina.pavlovskaja@hs-furtwangen.de

Dennis Maier

de.maier@hs-furtwangen.de

Stephanie Schäfer

stephanie.schaefer@hs-furtwangen.de

Ulrike Salat

ulrike.salat@hs-furtwangen.de

1 Faculty Medical and Life Sciences, Hochschule Furtwangen University, Campus Villingen-Schwenningen, Jakob-Kienzle-Str. 17, 78054 Villingen-Schwenningen, Germany

2 University Distillery, Hochschule Furtwangen University, Villingen-Schwenningen, Germany

3 Organic and Bioorganic Chemistry Lab, Hochschule Furtwangen University, Villingen-Schwenningen, Germany the process, modern methods for the monitoring of the fermentation and for the quality control of the final product are essential for the commercial success of the product. Various substances like ethanol and carbohydrates need to be quantified or, in the case of toxic substances like methanol, acetaldehyde, and ethyl acetate, excluded or quantified in relation to critical values.

In the field of food analytics, we find various methodologies for the determination of alcohol and volatile by-products in alcoholic drinks and spirits. There are official methods which are defined by corresponding regulations ("COMMISSION REGULATION (EC) No 2870/2000 of 19 December 2000 laying down Community reference methods for the analysis of spirits drinks," 2000) which include standard methods like pycnometry, electronic and hydrostatic balance based densimetry, and distillative methods and for which different standard operating procedure collections are available (Schmitt 1983; Tanner and Brunner 1987).

But also modern, mainly instrumentally based, methodologies have been developed by various groups over the past two decades, including near-infrared derivative spectrometry (Gallignani et al. 1993), high-performance liquid chromatography (Yarita et al. 2002), gas chromatography (Wang et al. 2003), and high-field ${ }^{1} \mathrm{H}$ NMR spectroscopy (Kew et al. 2017; Zuriarrain et al. 2015). The last one is a powerful tool, which allows qualitative and quantitative analyses of various substances like the ones involved in the fermentation process mentioned above, though in the case of mixtures the 
quantification can be challenging. Nonetheless, ${ }^{1} \mathrm{H}$ NMR has become an interesting analytical method used in various scientific fields beyond structural chemical analysis such as determination of blood alcohol concentration (Zailer and Diehl 2016) and quantitative metabolomics and metabolic profiling (Larive et al. 2015) and of course in food sciences (Hatzakis 2019).

One great advantage of magnetic resonance spectroscopy in the field of quantitative analysis is the fastness of the method. Once a standard operating procedure has been established, the analysis itself can be performed in under 15 min including sample preparation, measurement, and evaluation. On the other hand, a great disadvantage of high-field NMR is the high costs for the acquisition of the instrument as well as the following maintenance costs including personal, liquid nitrogen (weekly), and liquid helium (twice the year for modern magnets) for the cooling of the superconducting magnet.

Coming more from the field of teaching, the lowfield benchtop NMR technology started establishing itself in the field of scientific research over the past 5 years thanks to various meanwhile available machines on the market with ${ }^{1} \mathrm{H}$ frequencies ranging from 43 up to $100 \mathrm{MHz}$. These modern machines work with permanent magnets which are practically maintenance free and are delivered with a solid working basic software allowing to perform various $1 \mathrm{D}$ and $2 \mathrm{D}$ methods (if a second nuclei such as ${ }^{13} \mathrm{C}$ is available) (Blümich 2019; Blümich and Singh 2018; Lawson et al. 2020).

Various publications of the past few years show the growing importance and indicate clearly how benchtop NMR spectroscopy establishes itself in interdisciplinary scientific research (Antonides et al. 2019; Gunning et al. 2018; Jakes et al. 2015).

\section{Material and Methods}

Chemicals and reagents were purchased from Carl Roth and were used without further purification. For the determination of ethanol, two different test kits have been used, the "Enzytec Liquid Ethanol" from R-Biopharm — an NAD ${ }^{+}$alcohol dehydrogenase oxidation based method where the absorption of NADH is used to determine the ethanol concentration-and the "Ethanol UV Test" from R-Biopharm and Roche Diagnostics, which uses an additional oxidation step with aldehyde dehydrogenase to oxidize acetaldehyde to acetic acid. Both test kits work with the photometric determination of NADH.

\section{Preparation of the TSP- $d_{4} D_{2} O$ Solution}

$430.6 \mathrm{mg}$ of 3-(trimethylsilyl)-2,2,3,3-tetradeuteropropionic acid sodium salt $\left(\right.$ TSP- $\mathrm{d}_{4}$ ) has been dissolved in $30 \mathrm{ml} \mathrm{D} 2 \mathrm{O}$ and filled up to a volume of $50 \mathrm{ml}$ with $\mathrm{D}_{2} \mathrm{O}$ resulting in a $50 \mathrm{mM} \mathrm{TSP}^{-\mathrm{d}_{4}}$ solution in $\mathrm{D}_{2} \mathrm{O}$.

\section{NMR Sample Preparation and General Procedure for NMR Measurements}

For sample preparation, 10-ml samples of the fermentation mixture have been centrifuged yielding in a clear solution, which has been heated to $80{ }^{\circ} \mathrm{C}$ in a sealed tube for $15 \mathrm{~min}$ to stop enzymatic activities. After cooling to room temperature, the tube was shaken for $60 \mathrm{~s}$ to homogenize the solution. $100 \mu \mathrm{l}$ of this solution and $400 \mu \mathrm{l}$ of the TSP- $\mathrm{d}_{4} \mathrm{D}_{2} \mathrm{O}$ solution have been mixed and placed in a $5-\mathrm{mm}$ standard NMR tube resulting in a TSP- $\mathrm{d}_{4}$ standard concentration of $40 \mathrm{mM}$, which has been used to determine the concentrations of unknown analyte in solution.

${ }^{1} \mathrm{H}$ NMR spectra were recorded on a Magritek Spinsolve 60 Carbon Benchtop NMR spectrometer. Chemical shifts are reported in ppm relative to solvent signal (HDO: $\delta \mathrm{H}=$ $4.79 \mathrm{ppm}$ ). The spectra were recorded at a temperature of 25 ${ }^{\circ} \mathrm{C}$ with the 1D Proton standard protocol using 4 scans with a $90^{\circ}$ excitation pulse $(7 \mu \mathrm{s})$ covering a spectral range from 46 to $-37 \mathrm{ppm}$ (Magritek Spinsolve standard NMR conditions). $32 \mathrm{k}$ data points are acquired with an acquisition time of $6.5 \mathrm{~s}$ and a repetition time (recycle delay) of $15 \mathrm{~s}$. Data processing has been performed with MestReNova version 14.1.0 including zeroth and first-order phase correction and baseline correction with a Bernstein polynomial fit of third order.

\section{Test Kit Preparation and General Procedures}

The test kit preparation follows the general instructions from R-Biopharm and Roche Diagnostics available on the website of R-Biopharm (https://food.r-biopharm.com).

The preliminary preparation of the fermentation mixture follows the procedure described above (NMR sample preparation). Sample solutions with potentially higher concentrations have been diluted with deionized water. UV spectra have been recorded at $340 \mathrm{~nm}$ with a Shimadzu UV Visible Spectrophotometer UVmini-1240.

\section{Origin of the Barley Malt Mash and Sampling}

The wort - the liquid extracted from the mashing processhas been provided by the Badische Staatsbrauerei Rothaus AG. Commercially available Saccharomyces cerevisiae has been used for the fermentation process, from which 10 samples have been retrieved over a period of 4 days, after which the fermentation was completed. 


\section{Results and Discussion}

Before the start of the study, NMR spectra of pure glucose, saccharose, maltose, and ethanol have been measured under the standard procedure mentioned above to obtain spectral data for comparison. For both glucose and maltose, an anomeric mixture of alpha:beta $=36: 64$ could be found in equilibrium determined by integrating the anomeric doublets.

Figure 1 shows the NMR spectrum of the start of the fermentation process. Two doublets can be seen at 5.43 and 5.25 ppm, the first belonging to glycosidic anomeric protons of mainly maltose and also the glucosidic anomeric proton of saccharose which lies beneath this doublet. Together with the soluble dextrinic structures also present in the sample, the quantification of maltose becomes practically impossible.

The second singlet at $5.25 \mathrm{ppm}$ belongs to the alpha protons of glucose and of the reducing end of maltose, making it theoretically possible to determine the concentration of glucose and maltose in sum. Nonetheless, we decided to put this possibility aside because with proceeding fermentation, the concentration drops fast and the $S / N$ ratio declines rapidly under our standard procedure conditions resulting in unreliable results. Finally, no triplet can be seen at 1.18 showing that no ethanol has been produced so far.

Figure 2 shows the end of the fermentation process. Herein, only traces of remaining carbohydrates can be seen and a high amount of ethanol is detected without seeing relevant amounts of impurities like ethyl acetate or acetaldehyde. To calculate the concentration of ethanol in the sample, we calibrated the integral of the TSP- $\mathrm{d}_{4}$ signal at approx. 0 ppm to 9 , due to the nine protons of the trimethylsilyl group of the molecule.

After integrating the triplet at $1.18 \mathrm{ppm}$, which belongs to the methyl group of ethanol, the molar concentration can be determined by using following formula:

$c($ ethanol $)=\left(\mathrm{Int}_{\mathrm{met}} / 3\right) * c\left(\mathrm{TSP}-\mathrm{d}_{4}\right) *$ dilution-factor

Int $_{\text {met }}$ stands for the integration value of the triplet signal at $1.18 \mathrm{ppm}$, the TSP- $\mathrm{d}_{4}$ concentration in our case was adjusted to $40 \mathrm{mM}$, and the dilution factor is $5 / 1$. In the case of the NMR measurement shown in Fig. 2, an ethanol concentration can be calculated as shown below:

$$
(17,893 / 3) * 0.04 \mathrm{~mol} / 1 * 5=1.193 \mathrm{~mol} / 1
$$

For each sample, 4 measurements have been performed; the results are shown together with the values determined by the test kits in Table 1. Figure 3 shows the process of ethanol production in the barley malt mash graphically over time. Finally, Fig. 4 depicts the progress of ethanol production via a stacked NMR graphic showing nicely how the ethanol concentration increases, while the carbohydrate signals vanish from the spectra over time.

As displayed in Table 1 and in Fig. 3, the results of the test kits and from the NMR experiments match well. Interestingly,

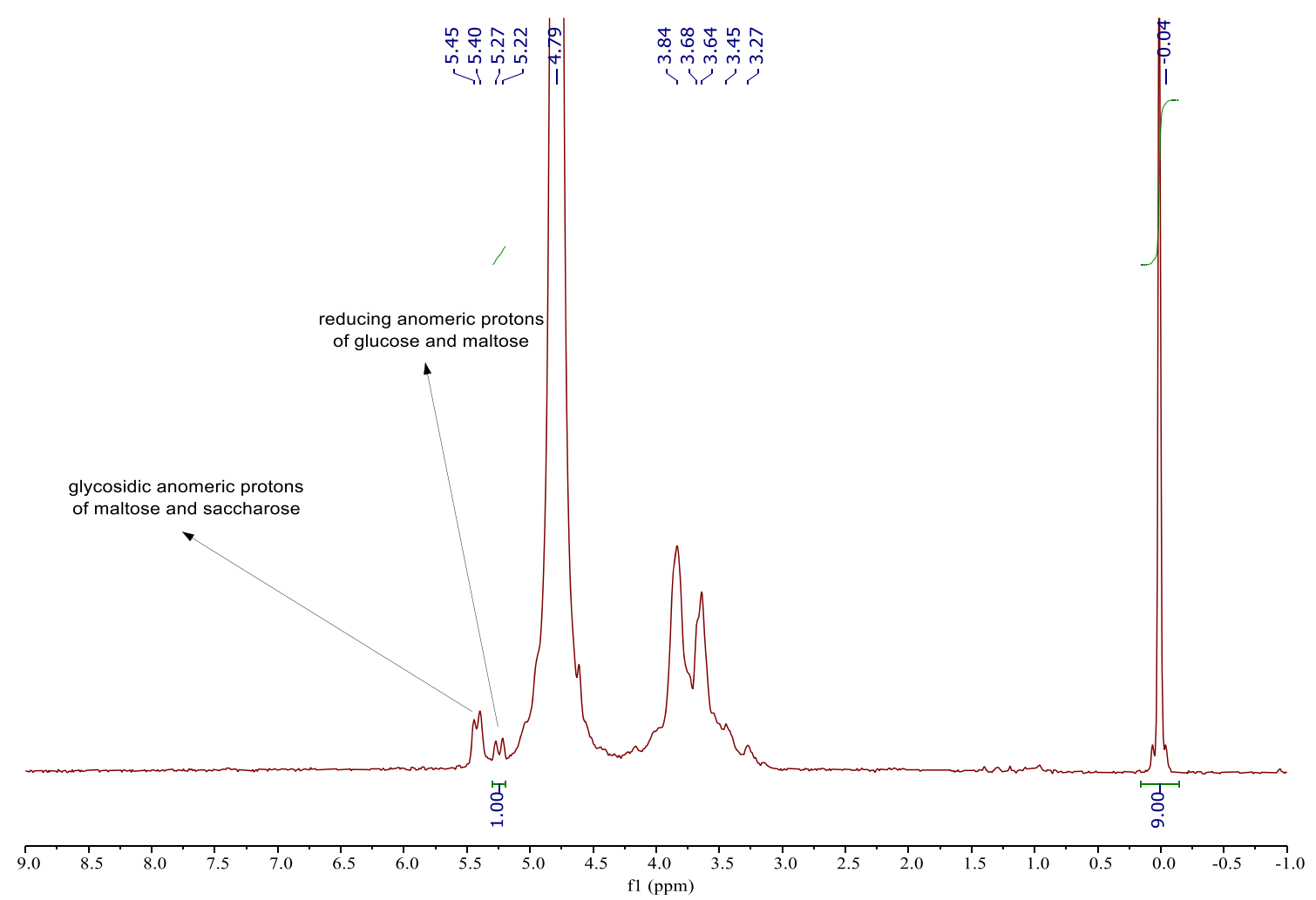

Fig. 1 Spectrum of the start of the fermentation process 


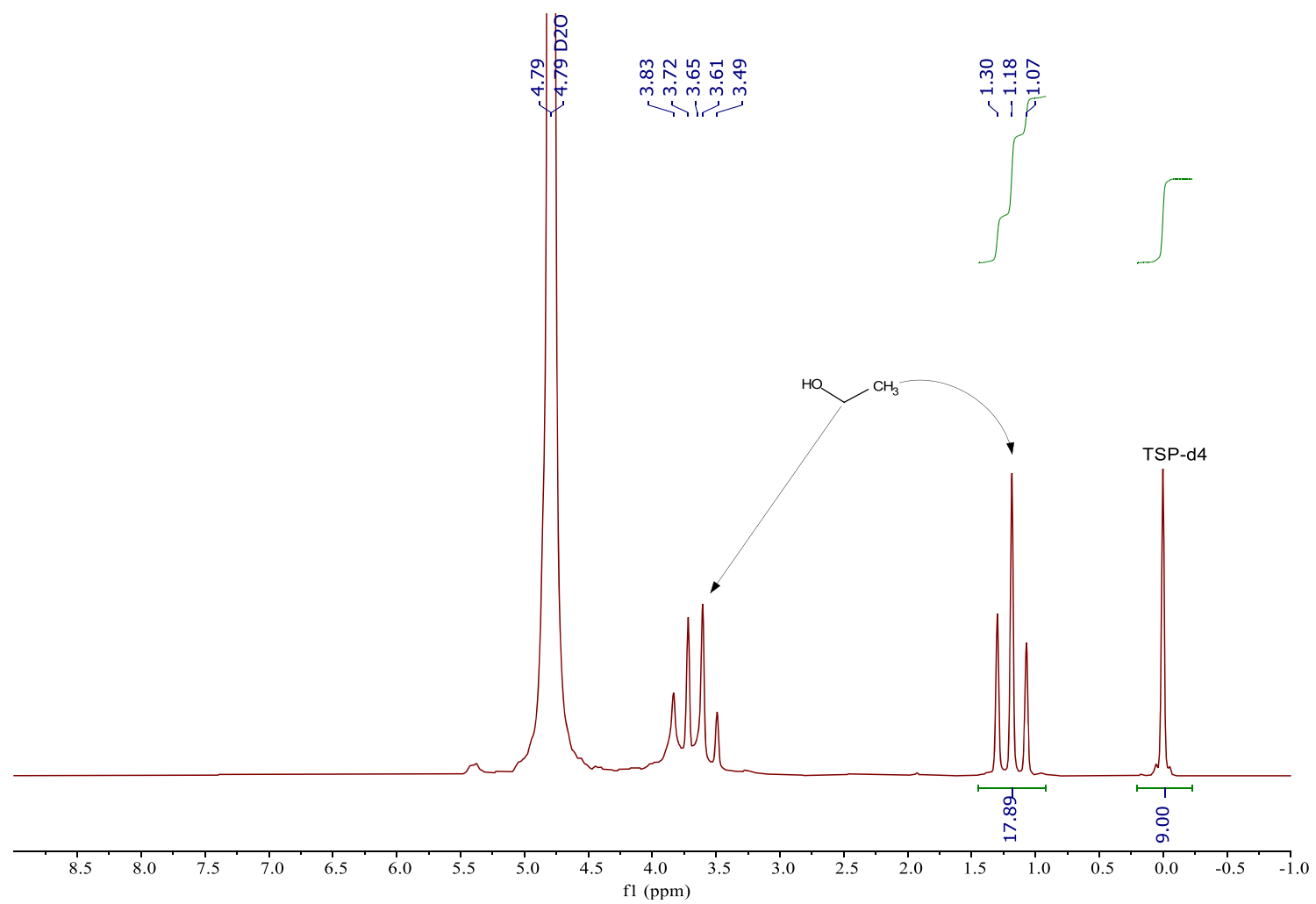

Fig. 2 Spectrum of the end of the fermentation process

both the NMR and the Enzytec Liquid Ethanol kit show a slight decrease in ethanol production between the hours 25 and 40 , though the effect is less distinct with the test kit.

More important, differences in the standard deviation (SD) can be observed for the different methods. While the NMR measurements show reproducible values resulting in a low SD, the Enzytec Liquid Ethanol test kit results in a broader distribution producing higher SD values and therefore a lower precision. This can partially be explained by the limitations of the enzymatic test kits. While it is theoretically and practically possible to determine every possible mixing ratio of ethanol and water by NMR spectroscopy, the measurement range for the enzymatic test kits given by the manufacturer's instructions lies between 20 and $300 \mathrm{mg} / \mathrm{l}$. This means that additional dilution steps are potentially necessary to get the samples into the recommended range, which leads to a second disadvantage of the test kits compared to the NMR spectroscopy: the sample preparation, which is in case of the NMR spectroscopy much faster and easier resulting in a lower chance for operational errors.
Table 1 Ethanol concentration obtained by the various quantification methods

\begin{tabular}{lllllll}
\hline Time (h) & $\begin{array}{l}c(\mathrm{~mol} / \mathrm{l}) \\
\text { NMR }\end{array}$ & SD & $\begin{array}{l}c(\mathrm{~mol} / \mathrm{l}) \\
\text { Enzytec Liquid Ethanol }\end{array}$ & $\begin{array}{l}c(\mathrm{~mol} / \mathrm{l}) \\
\text { Ethanol UV Test* }\end{array}$ \\
\hline 0 & 0.00 & \pm 0.0000 & 0.01 & \pm 0.0012 & 0.01 & nd \\
4.5 & 0.02 & \pm 0.0023 & 0.01 & \pm 0.0066 & 0.01 & nd \\
13 & 0.34 & \pm 0.0087 & 0.33 & \pm 0.0290 & 0.28 & nd \\
17 & 0.48 & \pm 0.0134 & 0.44 & \pm 0.0065 & 0.40 & nd \\
23 & 0.66 & \pm 0.0282 & 0.47 & \pm 0.0723 & 0.49 & nd \\
36 & 0.73 & \pm 0.0206 & 0.69 & \pm 0.0397 & 0.85 & nd \\
40 & 0.89 & \pm 0.0258 & 0.77 & \pm 0.0520 & 0.86 & nd \\
46 & 1.06 & \pm 0.0312 & 0.93 & \pm 0.0502 & 0.93 & nd \\
84 & 1.16 & \pm 0.0313 & 1.18 & \pm 0.1662 & 1.07 & nd \\
93 & 1.17 & \pm 0.0357 & 1.16 & \pm 0.2129 & 1.05 & nd \\
\hline
\end{tabular}

*Due to availability problems, only two measurements could be realized by the UV test, and as a result, no standard deviation could be determined 


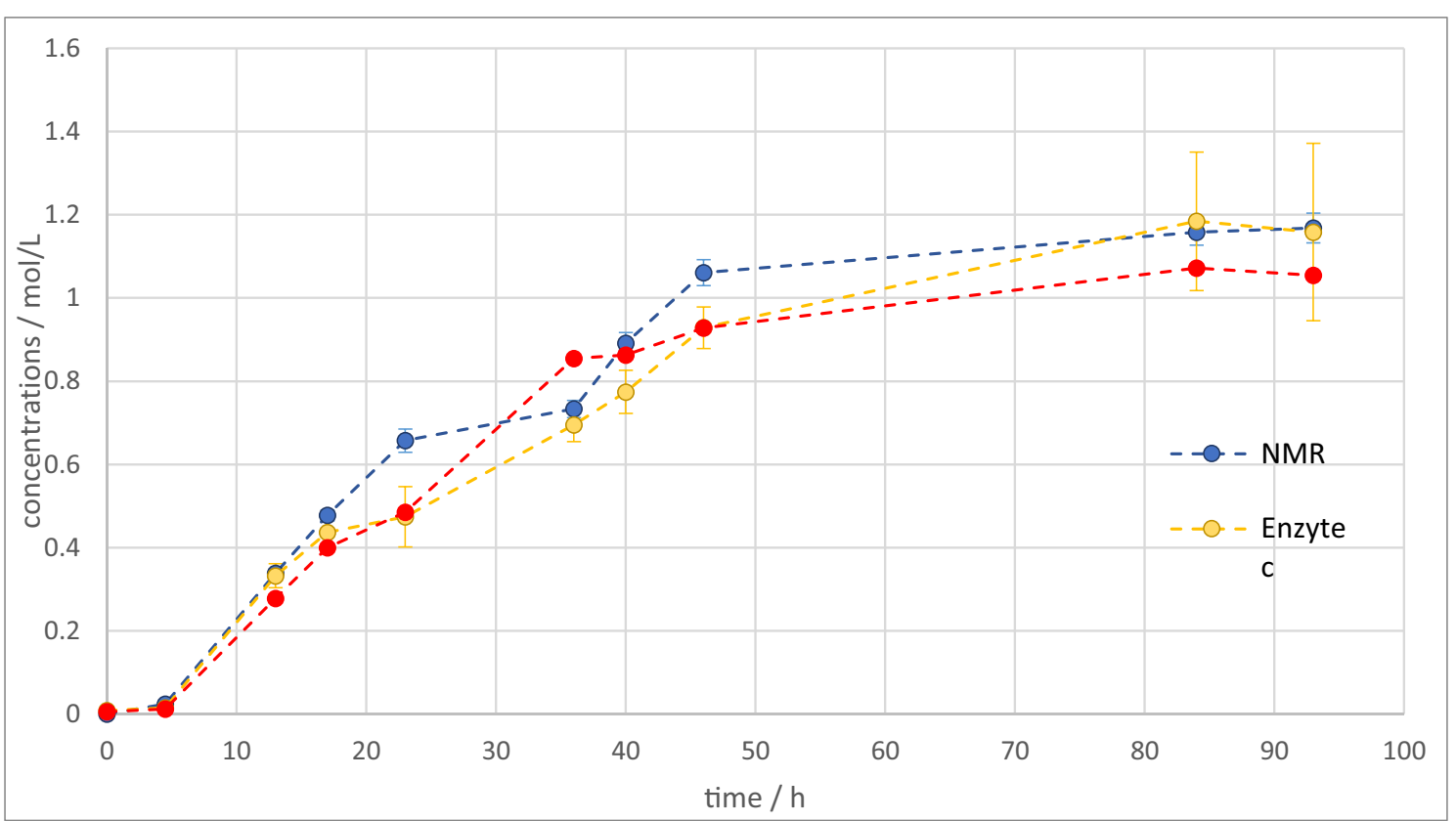

Fig. 3 Monitoring of ethanol production over time

On the first sight, the comparison of costs for the different methods is not easy. Though benchtop NMR spectrometers are much cheaper than high-field spectrometers and have most notably practically no maintenance costs, it is still a noteworthy value necessary for purchasing such a device. In light of this, an NMR spectrometer is used for a variety of applications from the determination of chemical structures of small molecules up to reaction kinetics. Furthermore, the

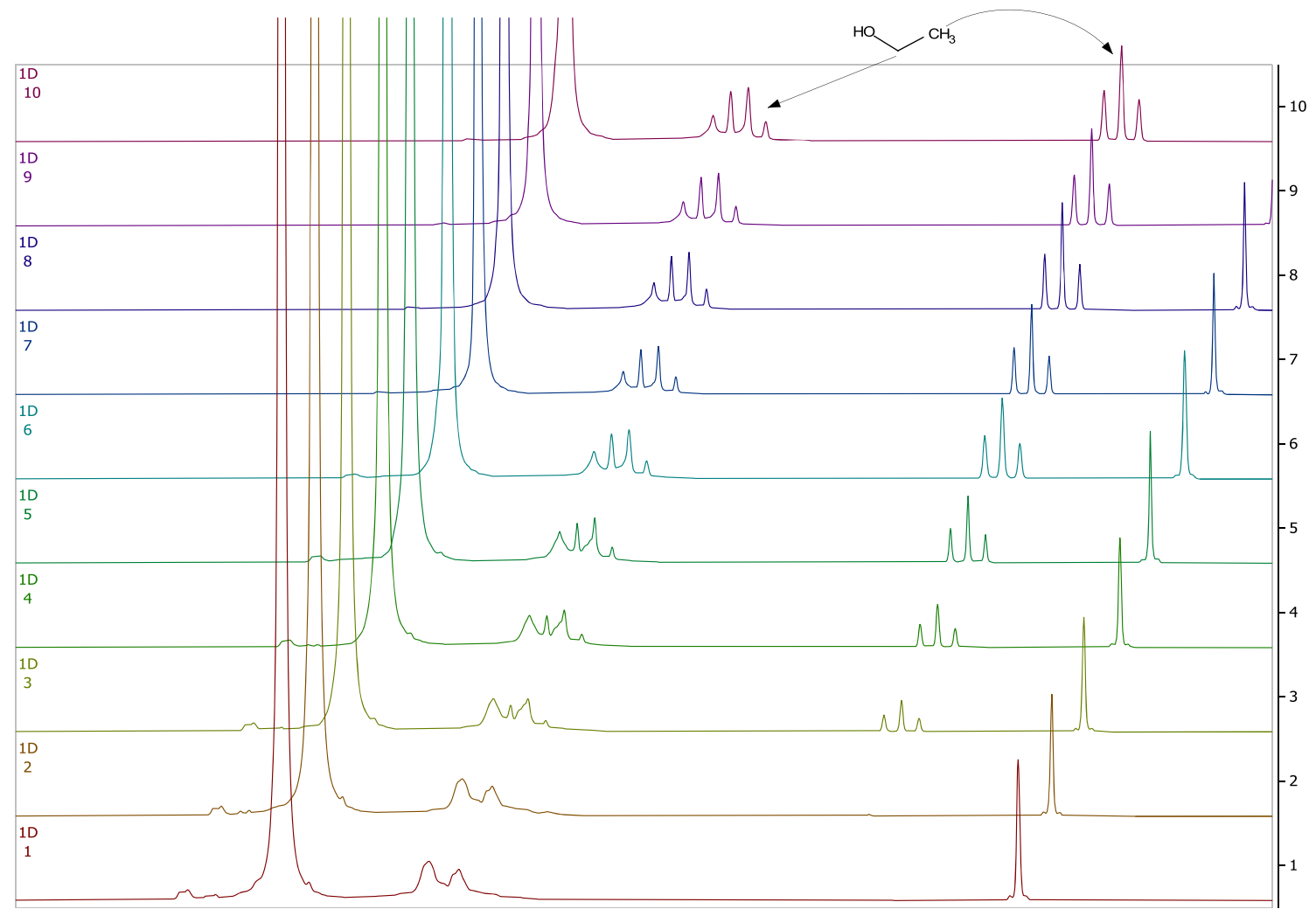

Fig. 4 Stacked NMR spectra of the time resolved fermentation process, spectrum 1 showing the start and spectrum 10 the end of the process 
timesaving workflow combined with the low follow-up costs (Magritek benchtop spectrometers do not necessarily need deuterated solvents due to an internal deuterium lock standard and NMR tubes can be used multiple times) must be considered an advantage. Based on this, the use of NMR spectroscopy for the presented purpose will over time be a time- and cost-saving alternative to enzymatic test kits. An economic quantification of this advantage is, nevertheless, not easy and depends significantly on the amount of measurements over time.

In terms of the quantification of the carbohydrates with NMR, as mentioned above, more work and method development is necessary to overcome some present limitations. One possibility would be to freeze dry the samples to get rid of volatile components and water. Then, NMR spectroscopy, i.e. with DMSO-d6 as solvent, could provide more and especially quantifiable information.

\section{Conclusion}

In this work, we were able to validate benchtop NMR spectroscopy as a method for the quantification of ethanol in a malt mash und compared the results with those of enzymatic test kits in terms of time, costs, applicability and accuracy. We could show that the results were at least comparable or better for the benchtop NMR-based quantification. Finally, we discussed the possibility to quantify other substances of the mash such as carbohydrates but had to admit that more research and work are required, to get reliable results.

Acknowledgements We are grateful to the Fonds der Chemischen Industrie for their financial support and to the Badische Staatsbrauerei Rothaus AG for providing the liquid extract of the mashing process (wort).

Funding Open Access funding enabled and organized by Projekt DEAL. The acquisition of the benchtop NMR spectrometer has been financially supported by the "Fonds der chemischen Industrie", part of the "Verband der chemischen Industrie", as a new tool for teaching and research.

\section{Declarations}

Ethical Approval This article does not contain any studies with human participants or animals performed by any of the authors.

Conflict of Interest Pia Burkhardtsmaier declares that she has no conflict of interest. Kristina Pavlovskaja declares that she has no conflict of interest. Dennis Maier declares that he has no conflict of interest. Stephanie Schäfer declares that she has no conflict of interest. Ulrike Salat declares that she has no conflict of interest. Magnus S. Schmidt declares that he has no conflict of interest.

Informed Consent Informed consent not applicable
Open Access This article is licensed under a Creative Commons Attribution 4.0 International License, which permits use, sharing, adaptation, distribution and reproduction in any medium or format, as long as you give appropriate credit to the original author(s) and the source, provide a link to the Creative Commons licence, and indicate if changes were made. The images or other third party material in this article are included in the article's Creative Commons licence, unless indicated otherwise in a credit line to the material. If material is not included in the article's Creative Commons licence and your intended use is not permitted by statutory regulation or exceeds the permitted use, you will need to obtain permission directly from the copyright holder. To view a copy of this licence, visit http://creativecommons.org/licenses/by/4.0/.

\section{References}

Antonides LH, Brignall RM, Costello A, Ellison J, Firth SE, Gilbert N, Groom BJ, Hudson SJ, Hulme MC, Marron J, Pullen ZA, Robertson TBR, Schofield CJ, Williamson DC, Kemsley EK, Sutcliffe OB, Mewis RE (2019) Rapid Identification of Novel Psychoactive and Other Controlled Substances Using Low-Field 1H NMR Spectroscopy. ACS Omega 4(4):7103-7112. https://doi.org/10. 1021/acsomega.9b00302

Blümich B (2019) Low-field and benchtop NMR. J Magn Reson 306:2735. https://doi.org/10.1016/j.jmr.2019.07.030

Blümich B, Singh K (2018) Desktop NMR and its applications from materials science to organic chemistry. Angew Chem Int Ed Engl 57(24):6996-7010. https://doi.org/10.1002/anie.201707084

COMMISSION REGULATION (EC) No 2870/2000 of 19 December 2000 laying down Community reference methods for the analysis of spirits drinks (2000). Official Journal of the European Communities. (L333), 20-46

Gallignani M, Garrigues S, La Guardia Md (1993) Direct determination of ethanol in all types of alcoholic beverages by near-infrared derivative spectrometry. Analyst 118(9):1167. https://doi.org/10.1039/ an9931801167

Gately I (2009) Drink: a cultural history of alcohol (1. ed., 1. paperback print). Gotham Books, New York

Gunning Y, Defernez M, Watson AD, Beadman N, Colquhoun IJ, Le Gall G et al (2018) 16-O-methylcafestol is present in ground roast Arabica coffees: Implications for authenticity testing. Food Chem 248:52-60. https://doi.org/10.1016/j.foodchem.2017.12.034

Hames G (2014) Alcohol in world history. themes in world history. Taylor and Francis, Hoboken Retrieved from http://gbv.eblib.com/ patron/FullRecord.aspx?p=1770565

Hatzakis E (2019) Nuclear magnetic resonance (NMR) spectroscopy in food science: a comprehensive review. Compr Rev Food Sci Food Saf 18(1):189-220. https://doi.org/10.1111/1541-4337.12408

Jakes W, Gerdova A, Defernez M, Watson AD, McCallum C, Limer E, Colquhoun IJ, Williamson DC, Kemsley EK (2015) Authentication of beef versus horse meat using $60 \mathrm{MHz} 1 \mathrm{H}$ NMR spectroscopy. Food Chem 175:1-9. https://doi.org/10.1016/j.foodchem.2014.11. 110

Kew W, Bell NGA, Goodall I, Uhrín D (2017) Advanced solvent signal suppression for the acquisition of 1D and 2D NMR spectra of Scotch Whisky. Magn Reson Chem 55(9):785-796. https://doi. org $/ 10.1002 / \mathrm{mrc} .4621$

Larive CK, Barding GA, Dinges MM (2015) Nmr spectroscopy for metabolomics and metabolic profiling. Analyt Chem 87(1):133-146. https://doi.org/10.1021/ac504075g

Lawson IJ, Ewart C, Kraft A, Ellis D (2020) Demystifying NMR spectroscopy: Applications of benchtop spectrometers in the 
undergraduate teaching laboratory. Magn Reson Chem. https://doi.org/10.1002/mrc.5055 Advance online publication

Phillips R (2014) Alcohol: A History. The University of North Carolina Press, Chapel Hill Retrieved from http://gbv.eblib.com/patron/ FullRecord.aspx?p=1663561

Tanner H, Brunner HR (1987) Getränke-Analytik: Untersuchungsmethoden für die Labor- und Betriebspraxis (2., neubearb. u. erw. Aufl.). Verl. Heller Chemie- u. Verwaltungsges, Schwäbisch Hall

Tanner H, Brunner HR (1987) Getränke-Analytik: Untersuchungsmethoden für die Labor- und Betriebspraxis (2., neubearb. u. erw. Aufl.). Verl. Heller Chemie- u. Verwaltungsges, Schwäbisch Hall

Wang M-L, Choong Y-M, Su N-W, Lee M-S (2003) A rapid method for determination of ethanol in alcoholic beverages using capillary gas chromatography. J Food Drug Anal 11(2):10.38212/222410.36614.2710
Yarita T, Nakajima R, Otsuka S, Ihara T, Takatsu A, Shibukawa M (2002) Determination of ethanol in alcoholic beverages by highperformance liquid chromatography-flame ionization detection using pure water as mobile phase. J Chromatogr A 976(1-2):387391. https://doi.org/10.1016/S0021-9673(02)00942-1

Zailer E, Diehl BWK (2016) Alternative determination of blood alcohol concentration by (1)H NMR spectroscopy. J Pharm Biomed Anal 119:59-64. https://doi.org/10.1016/j.jpba.2015.11.030

Zuriarrain A, Zuriarrain J, Villar M, Berregi I (2015) Quantitative determination of ethanol in cider by $1 \mathrm{H}$ NMR spectrometry. Food Control 50:758-762. https://doi.org/10.1016/j.foodcont.2014.10. 024

Publisher's Note Springer Nature remains neutral with regard to jurisdictional claims in published maps and institutional affiliations. 